\title{
MORTALIDADE MATERNA: AS EVIDÊNCIAS CIENTÍFICAS COMO INSTRUMENTO DE INTERVENÇÃO
}

\author{
MATERNAL MORTALITY: SCIENTIFIC EVIDENCE AS AN INTERVENTION \\ INSTRUMENT
}

\author{
Raquel Maria de Jesus Souza Cunha ${ }^{1}$ \\ Maria Janaílda Araújo Furtado ${ }^{2}$ \\ Lucélia Soares da Silva ${ }^{3}$
}

RESUMO: A mortalidade materna constitui-se um grave problema de saúde pública, sendo os países em desenvolvimento os que apresentam maior número de mortes por nascidos vivos, assim como o Brasil que registra uma taxa correspondente a 6o mortes para cada Ioo mil nascidos vivos, enquanto nos países desenvolvidos esses dados são menores, contabilizando 20 mortes para cada cem mil nascidos vivos. No cenário brasileiro as maiores taxas de mortalidade materna estão registradas nos Estados do Piauí e Maranhão, provocando nas equipes de saúde a importância de buscar medidas que visem a redução deste quadro. Objetivos: Identificar a partir das evidências científicas as principais estratégias de prevenção da mortalidade materna. Método: Revisão Integrativa da Literatura. Resultado: A má condução do pré-natal na atenção básica é apontada como fator determinante e intrínseco no desfecho negativo da realidade da mortalidade materna. Conclusão: $\mathrm{O}$ estudo contribuiu significativamente para a análise da mortalidade materna e as estratégias de redução a partir das evidências científicas.

Palavras-chave: Mortalidade materna. Prática clínica baseada em evidências. Política de saúde.

ABSTRACT: Maternal mortality is a serious public health problem, with developing countries having the highest number of deaths by live births, as well as Brazil, which has a rate corresponding to 60 deaths for every roo,ooo live births, while in developed countries these figures are lower, accounting for 20 deaths for every hundred thousand live births. In the Brazilian scenario, the highest maternal mortality rates are registered in the States of Piauí and Maranhão, provoking in the health teams the importance of seeking measures aimed at reducing this situation. Objectives: To identify, based on scientific evidence, the

\footnotetext{
I Profissional de Educação Física/Docente do Curso de Educação Física da Universidade Paulista UNIP/Especialista em Saúde e Comunidade- UFPI/Mestre em Saúde da Mulher - UFPI. E-mail: rmedf8i@hotmail.com.

${ }^{2}$ Enfermeira/Diretora administrativa do Hospital Estadual Dirceu Arco Verde de Parnaíba -PI/ Especialista em Gestão da clínica - Hospital Albert Einstein/Mestre em Saúde da Mulher - UFPI. E-mail: janaildafurtado@hotmail.com.

3 Enfermeira/Gerente da Estratégia de Saúde da Família do município de Parnaíba - PI/Especialista em educação na saúde para preceptoria no SUS/Mestre em Saúde da Mulher - UFPI. E-mail: luceliaog@hotmail.com.
} 
main strategies for the prevention of maternal mortality. Method: Integrative Literature Review. Result: Poor prenatal care in primary care is identified as a determining and intrinsic factor in the negative outcome of the reality of maternal mortality. Conclusion: The study contributed significantly to the analysis of maternal mortality and reduction strategies based on scientific evidence.

keywords: Maternal mortality. Evidence-based clinical practice. Health policy health.

\section{INTRODUÇÃO}

Segundo a Organização Mundial de Saúde (OMS) morte materna é a morte de uma mulher durante a gestação, ou dentro de um período de 42 dias após o término da gestação, independentemente da duração ou da localização da gravidez, devido a qualquer causa relacionada com a gravidez ou agravada pela mesma, ou a medidas tomadas em relação a ela, porém não devido a causas acidentais ou incidentais. (MINISTÉRIO DA SAÚDE, 2009).

A morte materna não é mais considerada fatalidade e sua análise passaram a marcar o desenvolvimento da população, uma vez que 99\% delas acontecem nos países em desenvolvimento e por causas evitáveis com melhoria no acesso e qualidade dos serviços de saúde (SZWARCWALD CL et al, 2014). Quando se fala em causas evitáveis está relacionando com situações em que as mortes não deveriam ter ocorrido (FERRAZ; BONDGNON, 2012).

A estimativa da razão de morte materna (RMM) nos países desenvolvidos é de 20 mortes por 100.000 nascido vivos e 440/100.00o nos países em desenvolvimento. Nos Estados Unidos e Canadá a razão é inferior a 9/10o.ooo nascidos vivos, grande disparidade em relação ao Brasil que em 1990 era 140 mortes/Ioo.ooo nascidos vivos. Houve redução importante registrando 75/100.000 em 2007, mas ainda muito distante da realidade dos países desenvolvidos (FRANCISCO, ET AL, 2013).

As causas de morte materna podem estar diretamente ou indiretamente ligadas a gravidez e parto. A razão de morte por causas diretas em relação as indiretas reduziram de 9.4 para 3,2 no mesmo período devido melhora nas investigações de mortes de mulheres em idade fértil (MIF), isso pode também características variáveis em relação ao local de ocorrência, é o que mostra seu estudo onde faz uma análise comparativa entre os comitês de mortalidade materna de São Paulo e Paraná em relação a causa secundária, encontrando 
as complicações do aborto a principal causa de morte materna no estado de São Paulo, já no Paraná, Pré-eclâmpsia/eclampsia e complicações do parto(IPEA, 2019).

Diante desse elevado quantitativo global e desproporcional de óbitos maternos nos diferentes níveis de desenvolvimento dos países, a Organização das Nações Unidas (ONU) definiu em reunião com governantes de 189 países no ano 2000 os Objetivos de Desenvolvimento do Milênio (ODM) que tinham como quinta meta reduzir a mortalidade materna em $3 / 4$ até 2015. Apesar dos esforços o Brasil não chegou a meta proposta que era 35 mortes maternas por 100.000 nascimentos, registrando em 2015 razão de mortalidade materna superior a 6o mortes por Ioo.ooo nascidos vivos. (GUARDA, 2017, p. 15).

Essa heterogeneidade na taxa de mortalidade materna é observada também comparando as regiões do Brasil, sendo a maior incidência na região nordeste (80/10o.ooo) e os estados que apresentam os piores indicadores são o Piauí (ıor,8/10o.ooo) e Maranhão (II4/IOo.ooo) segundo dados levantados em zori quando a mortalidade do Brasil registrava 64,8/10o.ooo nascidos. (MADEIRO, 2018, p.I)

O presente estudo traz relevante contribuição para trabalhos posteriores bem como referência para os serviços de saúde adotarem práticas baseadas em evidências científicas para redução da mortalidade materna.

\section{Método: Revisão Integrativa da Literatura (RIL)}

Inicialmente para elaboração da pergunta de pesquisa foi utilizada a estratégia PICO - Estratégia para elaboração da pergunta de pesquisa (acrômio para P: população/pacientes; I: Interesse; C: Intervenção; O: Desfecho) de acordo com quadro abaixo

\begin{tabular}{|l|l|}
\hline P & Mortalidade materna \\
\hline $\begin{array}{l}\text { I } \\
\text { Interesse }\end{array}$ & Cuidados em saúde e prevenção \\
\hline $\begin{array}{l}\text { C } \\
\text { Intervenção }\end{array}$ & Evidências científicas \\
\hline $\begin{array}{l}\text { O } \\
\text { Desfecho }\end{array}$ & Redução da mortalidade materna \\
\hline
\end{tabular}


Pergunta norteadora: Quais as melhores intervenções disponíveis nas evidências científicas para evitar a mortalidade materna?

Protocolos e registros: Conduziu-se conforme a metodologia Preferred Reporting Items for Systematic Reviews and Meta-Analyses (PRISMA).

Seleção dos estudos: Foram utilizados termos em inglês e português para a inclusão dos artigos, empregando os seguintes critérios: estudos de revisão sistemática, relato de casos, estudo de coorte, Guia de prática clínica, estudo de casos e controles e ensaio clínico controlado, cujos desfechos eram estratégias de redução da mortalidade materna publicados nos últimos cinco anos nas Bases de dados MEDLINE e LILACS.

Fontes de informações: Para identificar os artigos acerca do assunto, realizou-se busca na BVS (Biblioteca Virtual de Saúde) nas bases MEDLINE e LILACS, de janeiro a março de 2020 .

Estratégia de busca: Estratégias de redução da mortalidade materna: (db:("MEDLINE" OR "LILACS") AND mj:("Mortalidade Materna") AND type_of_study:("case_reports" OR "cohort" OR "guideline" OR "case_control" OR "systematic_reviews" OR "clinical_trials") AND la:("en" OR "pt")) AND (year_cluster:[2015 TO 2019]).

Processo de coleta de dados: Após a consulta às bases de dados e a aplicação da estratégia de busca, foram identificados estudos que apresentavam duplicidade entre as bases. Foram lidos todos os resumos resultantes. Nos casos que a leitura do resumo não era suficiente para estabelecer se o artigo deveria ser incluído, considerando-se os critérios de inclusão definidos, o artigo foi lido na íntegra para determinar sua elegibilidade. Quando o resumo era suficiente, os artigos eram selecionados e então obtida a versão integral para confirmação de elegibilidade e inclusão no estudo. Para extração dos dados dos artigos, elaborou-se um instrumento contendo as seguintes informações: ano de publicação, autor, local de publicação, tipo de estudo, tamanho da amostra.

Critérios de exclusão: Foram excluídos os artigos que o assunto não tratava da temática proposta (estratégias de redução da mortalidade materna). 
Fluxograma de identificação e seleção dos artigos para revisão integrativa sobre causas e estratégias de redução da mortalidade materna publicados nas bases de dados MEDLINE E LILACS, 2015 A 2019.

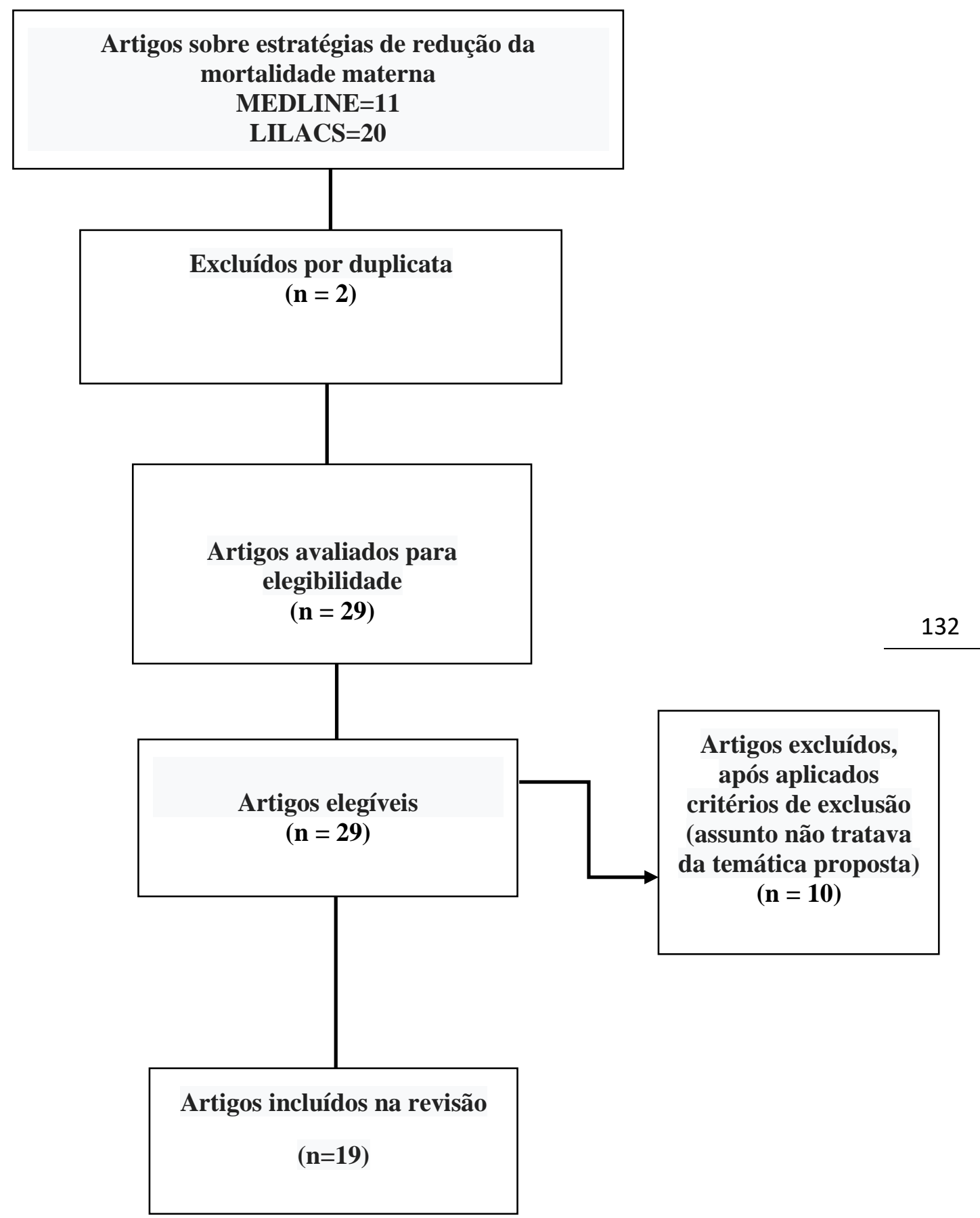

Resultados: Os resultados apresentados na pesquisa foram descritos através de análise estatística quantitativa e qualitativa, processados e analisados no programa Microsoft Excel a partir da estatística descritiva (média). Na descrição foram apresentadas 
medidas absolutas ( $\left.\mathrm{n}^{\circ}\right)$ e relativas (\%), para análise das frequências e da distribuição dos dados.

\section{Características dos estudos e estratégias apontadas para redução da mortalidade materna entre os anos de 2015 a 2019.}

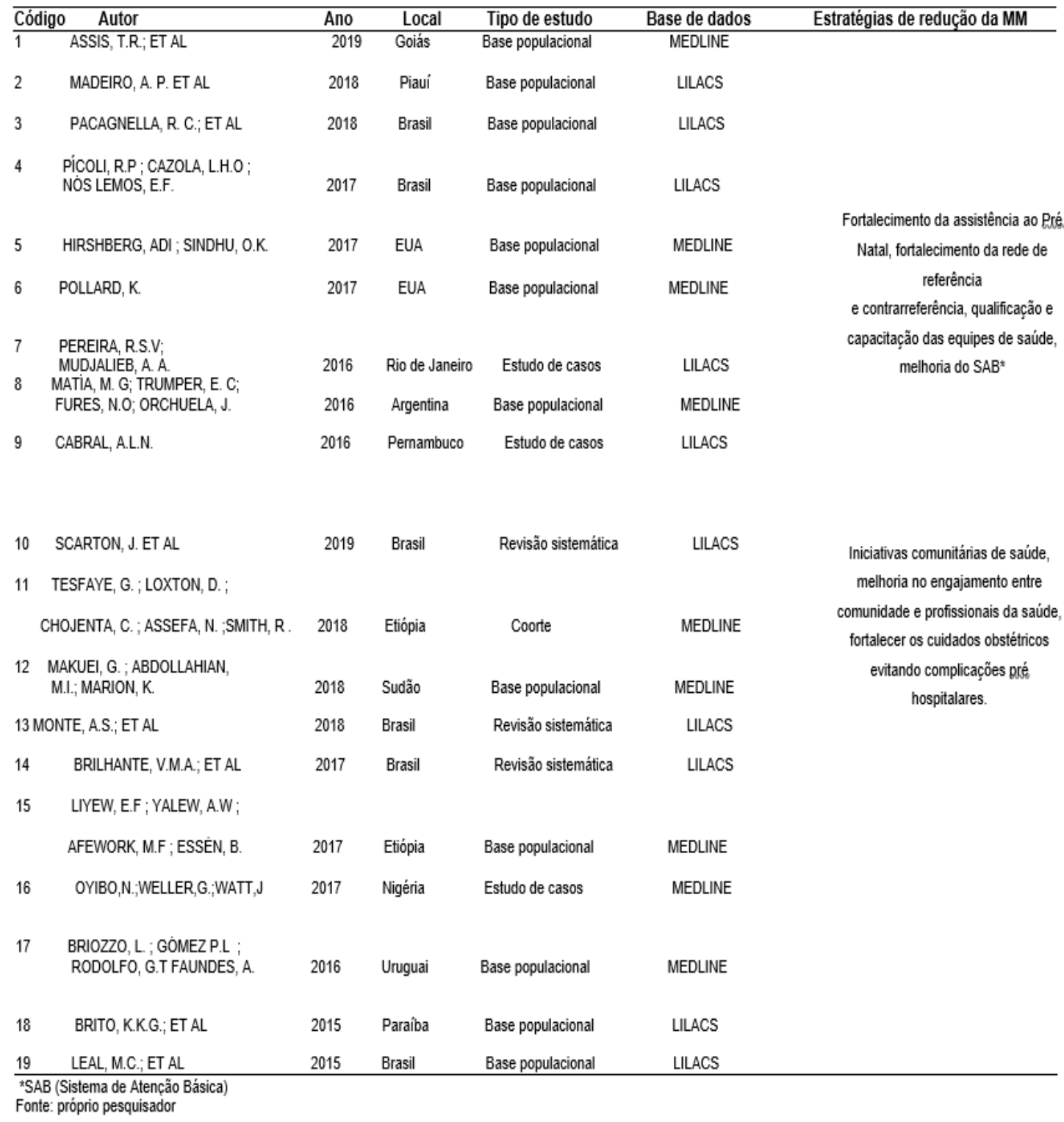

Os artigos selecionados para a revisão integrativa da literatura predominaram do tipo Base populacional, isso garantiu investigar além do objetivo proposto os determinantes sociais e biológicos de mortalidade materna. De acordo com Francisco, et al, (2013) esse tipo de estudo compõe importante instrumento para avaliar indicadores de 
qualidade de saúde, de procedimentos pertinentes à saúde, de promoção e uso de serviços de saúde, e de morbimortalidade de uma determinada população.

Outro dado importante a ser destacado é o local dos estudos que prevaleceu o Brasil, com um apanhado geral das regiões, trazendo uma maior aproximação dos resultados encontrados com a realidade proposta. Para Barros (2018) os levantamentos nacionais de saúde disponibilizam elementos para a avaliação e o acompanhamento das ações implementadas pelo Sistema Público de saúde promovendo a descentralização das ações pela competência de quantificar e qualificar fatores de risco alteráveis.

As bases de dados LILACS e MEDLINE destacadas na revisão contribuem significativamente na segurança dos achados por se tratar de bases que abarcam obras especializadas em saúde, com literatura técnica e de fácil acesso. Segundo Paker; Tardelli e Castro (2017), as bases de dados LILACS e MEDLINE são fontes de ciência que admitem aferir a produção científica nas diferentes áreas do conhecimento em saúde, avaliando suas qualidades e advertindo sua evolução ao longo dos anos nos distintos países.

Os estudos publicados recentemente entre os anos de 2015 e 2018 constituem as mais atuais recomendações que os serviços de saúde promovem, e abarcam desde a educação constante dos profissionais que atuam na atenção pré-natal, sobretudo no que se refere a comunicação com as gestantes, o correto manuseio das ações bem como a valorização dos instrumentos disponíveis para identificar o mais cedo, riscos relacionados ao ciclo gravídico-puerperal.

O fortalecimento do pré-natal foi apontado como um dos fatores que implicam diretamente na logística de ações preventivas e de combate à mortalidade materna na maioria dos estudos selecionados, e começa ressaltando ações de avaliação a saúde da mulher e do feto e seu desenvolvimento, avalizando o bem-estar; identificando fatores de risco e conduzindo a gestante para níveis de referência de superior complexidade que garantam tratamento precoce das condições de saúde afetadas.

O Caderno de atenção ao pré-natal do MS (Ministério da saúde) esclarece que a assistência adequada ao pré-natal favorece a qualificação do cuidado e a articulação das redes assistenciais envolvidas. Esse processo constitui-se em uma ferramenta que, incluída à aptidão das equipes e dos gestores, pode colaborar para o contínuo progresso do acesso e 
da qualidade dos desfechos. Para isso é imprescindível que essa ferramenta apareça efetiva (MINISTÉRIO DA SAÚDE, 2012).

A qualificação das equipes também foi registrada nos achados da pesquisa como instrumento eficaz na assistência à mulher na gravidez e puerpério provocando resultados satisfatórios no que tende a prevenir e reduzir agravos a saúde materna, contribuindo com a preservação da vida, uma vez que se o manuseio consciente e seguro das ações está intrinsicamente relacionado à capacitação pessoal e individual das equipes ligadas ao processo de cuidado.

O Ministério da saúde aborda que as equipes envolvidas nas ações que oferecem o cuidado a gestante e no puerpério devem estar constantemente capacitadas, desde a disposição da metodologia de trabalho, do serviço de saúde e aspectos do planejamento, além de questões relacionadas ao acompanhamento da gravidez de risco tradicional e de suas possíveis intercorrências, promoção da saúde, gestação em situações especiais, auxílio ao parto, até as questões legais (MINISTÉRIO DA SAÚDE, 2012).

A atenção primária à saúde, neste sentido, deve dispor de aparelhagem técnica e científica para melhor condução da assistência à mulher. A comunicação e a educação em saúde necessitam aparecer de forma efetiva no planejamento das ações.

Koffman e Bonadio (2019) destacam que a assistência pré-natal deve ser fortalecida a priori na atenção primária a saúde estabelecendo um conjunto de atividades que objetive promover a saúde da gestante e do feto, identificando os possíveis riscos decorrente do estágio gravídico e pós gravidez de forma individualizada. Ainda, avaliam que a carência e ou falha dessa logística fica comprovadamente conexa a maiores coeficientes de mortalidade materna.

Investir no progresso da qualidade da assistência pré-natal oferecida, na qualificação dos profissionais responsáveis pelo acolhimento da mulher ao longo do período gravídico-puerperal, bem como aperfeiçoar as estruturas físicas dos estabelecimentos de saúde que acompanham essas gestantes, tanto para a efetivação do pré-natal de baixo risco, de alto risco como para o atendimento ao parto e pós-parto, são determinadas das intervenções sugeridas na literatura estudada para prevenir e reduzir a mortalidade materna. 
Apesar dos numerosos acrescentamentos tecnológicos e científicos e de ser comprovadamente um óbito evitável, a mortalidade materna, permanece como amplo problema de saúde pública a ser afrontado. Porém, a identificação precoce de diferentes riscos obstétricos bem como a intervenção oportuna, permitem um melhor desfecho na direção a alocação de soluções imprescindíveis para evitar as complicações, em analogia aos riscos, estes só são identificados com a efetivação de um pré-natal de qualidade e eficaz, como confirmam as evidências (CARVALHO, ET AL, 2015).

De acordo com os achados na pesquisa o pré-natal bem realizado na atenção básica pode reduzir complicações no período gestacional e facilitar o desempenho dos especialistas durante o parto, colaborando na redução das infecções e riscos eminentes ao parto, além do acompanhamento da saúde da mãe e bebê.

Um acompanhamento pré-natal efetivo tem um amplo impacto na diminuição da mortalidade materna desde que as mulheres tenham acesso facilitado aos serviços, os quais devem ter qualidade aceitável para identificar precocemente os fatores de riscos a serem controlados oportunamente. Melhorar e ampliar a assistência à mulher aparece, portanto, como ponto de partida para reverter o quadro atual de mortalidade materna, com a reestruturação dos serviços de saúde e a qualificação dos profissionais que compõem desde a base à alta complexidade.

No âmbito público, algumas ações já foram significativamente implantadas como o Programa de Humanização no Pré-Natal e Nascimento (PHPN), que busca assegurar a melhoria do acesso, da cobertura e da qualidade do acompanhamento pré-natal, assim como da assistência ao parto e pós-parto, bem como, a criação do Pacto Nacional de Redução da Mortalidade Materna e Neonatal que estruturado as três esferas do governo em parceria com a sociedade civil (CARVALHO, ET AL, 2015).

\section{CONCLUSÃO}

A mortalidade materna ainda é um problema que causa grande impacto na família e norteia um importante indicador de saúde da mulher. Dessa forma o estudo buscou estratégias para redução dessa problemática baseadas em evidência científica com os fatores que implicam nesse desfecho desfavorável. 
O fortalecimento da assistência ao pré-natal, por meio da qualificação dos profissionais da Estratégia Saúde da Família para uma detecção precoce dos fatores de risco, encaminhamento e condução adequada no tratamento das intercorrências é o que o estudo encontrou nas evidências.

Recomenda-se estudos posteriores para avaliar os efeitos da capacitação e comportamento da mortalidade materna, bem como a importância dos comitês de óbito materno para identificar a extensão da mortalidade materna, os fatores que a determinam; e sugerir medidas que previnam novas mortes. Eles colaboram ainda para a melhoria da informação sobre o óbito materno, mostrando os resultados da assistência prestada às gestantes.

\section{REFERÊNCIAS}

ASSIS, T.R.; ET AL. Implementação da Rede Cegonha em uma Regional de Saúde do estado de Goiás: o que os indicadores de saúde mostram sobre atenção materno-infantil? Reciis - Rev Eletron Comun Inf Inov Saúde. 2019 out.-dez.;13(4):843-53 | [www.reciis.icict.fiocruz.br] e-ISSN 1981-6278, 2019

BARROS, M.B.A. Inquéritos domiciliares de saúde: potencialidades e desafios. Rev Bras Epidemiol.;II(supl I):6-19. DOI:10.1590/SI415-790X2008000500002, 2018.

BRASIL. IBGE. Censo Demográfico, 2016. Disponível em:〈http://www.ibge.gov.br〉. Acesso em: 12 março. 2018.

- Ministério da Saúde. Secretaria de Atenção à Saúde. Departamento de Ações Programáticas Estratégicas. Política nacional de atenção integral à saúde da mulher: princípios e diretrizes. Brasília: Editora do Ministério da Saúde, 2009.

- Ministério da Saúde. Secretaria de Atenção à Saúde. Departamento de Ações Programáticas Estratégicas. Política Nacional de Atenção a Saúde da Mulher, 2012. 
BRILHANTE, V. M.; ET AL. Maternal Near Miss as health care indicator: an integrative review. Rev. bras. promoç. saúde (Impr.) ; 30(4): I-9, 06/12/2017.

BRIOZZO, L. ; GÓMEZ, P. L., R. ; TOMASSO, G.; FAUNDES, A. Taxas de mortalidade materna geral e relacionadas ao aborto no Uruguai nos últimos 25 anos e sua associação com políticas e ações destinadas a proteger os direitos das mulheres. Int $\mathrm{J}$ Gynaecol Obstet ; $134\left(\mathrm{~S}_{1}\right)$ : S2O-S23, agosto de 2016.

BRITO, K.K.G; MOURA, J.R.P; SOUSA, M.J; BRITO, J.V ; OLIVEIRA, S.H. S.; SOARES, M.J.G.O. Prevalencia de las síndromes hipertensivas específicas del embarazo (GHS). Rev. pesqui. cuid. fundam. (Online) ; 7(3): 2117-2125, jul.-set. 2015.

CABRAL, A.L.N. Evaluation of the Impact of the Mother Owl Program in the rates of infant and maternal mortality in Pernambuco. ECOS | ID: biblio-988363; Recife; s.n; 2016.

CARVALHO, M. L. ET AL. Prevenção da mortalidade materna no pré-natal: uma revisão integrativa. R. Interd. v. 8, n. 2, p. 178-184, abr. mai. jun. 2015.

FERRAZ, L.; BONDIGNON, M. Mortalidade materna no Brasil: uma realidade que precisa melhorar. Revista Baiana de Saúde Pública. V 36, no 02, 2012.

FRANCISCO, P.M.S.B; BARROS, M. B. A.; SEGRI, N.J.; ALVES, M.C.G.P. Comparação de estimativas de inquéritos de base populacional. Rev. Saúde Pública vol.47 no.I São Paulo, 2013.

GUARDA, O. I. Mortalidade materna no Estado do Maranhão. Tedebc.ufma.br: https://tedebc.ufma.br/jspui/handle/tede/tede/1356, 2017.

HIRSHBERG, ADI; ABUSO E CENTRO DE SEGURANÇA, O K SINDHU. Epidemiologia da morbimortalidade materna. Semin Perinatol ; 4I (6): 332-337, 2017. 
IPEA. Instituto de Pesquisa Econômica Aplicada. Disponível na URL < https://www.ipea.gov.br/portal/index.php?option=com_content $\&$ view=article $\&$ id $=35013$ \&catid $=$ Io\&Itemid $=9>$ acesso em o9 de fevereiro de 2019 .

KOFFMAN, M.D.; BONADIO, I.C. Avaliação da atenção pré-natal em uma instituição filantrópica da cidade de São Paulo. Rev Bras Saúde Matern Infant, Recife, v. 5, n. I, p. 2332, 2019.

LEAL， M.C; THEME-FILHA， M.M; MOURA， E.C; CECATTI， J.G.; SANTOS, LEONOR M.P. Prenatal and childbirth care for women using the public health system resident in Amazonia Legal and the Northeast Region of Brazil 2oro. Rev. bras. saúde matern. infant ; 15(I): 91-104, Jan-Mar/2015.

LIYEW, E.F.; YALEW, ALEMAYEHU W.;AFEWORK, M.F.; ESSÉN, B. Incidência e causas de near miss materna em hospitais selecionados de Addis Abeba, Etiópia. PLoS One ; 12 (6): eo179013, 2017.

MADEIRO, A. P.; RUFINO, A. C.; NUNES, M. D.; QUEIROZ, I. C.; CARVALHO, K. R.; QUEIROZ, L. C.. Mortalidad de las mujeres en edad reproductiva en Piauí, Brasil, 2008-2012: causas básicas de las muertes y factores associados. Rev. epidemiol. controle infecç ; 8(4): 442-449, out.-dez. 2018.

MAKUEI, G. ; ABDOLLAHIAN, M. ; MARION, K. Limites de perfil ideal para a taxa de mortalidade materna (MMR) no Sudão do Sul. BMC Gravidez Parto ; I8 (I): 278, 2018.

MATÍA, M. G; TRUMPER, E. C; FURES, N. O.; ORCHUELA, J. Uma replicação do modelo uruguaio na província de Buenos Aires, Argentina, como política pública para reduzir a mortalidade materna relacionada ao aborto. Int J Gynaecol Obstet ; I34 ( $\mathrm{S}_{1}$ ): $\mathrm{S}_{31}$ S34, agosto de 2016. 
MONTE, A.S; MENDES, I.C.; ORIÁ, M.B.O.; CARVALHO, F.H.C; BROWN, H.; DAMASCENO, A.K.C. Near miss maternal: influencing factors and guidelines for reducing maternal morbidity and mortality. Rev Rene (Online) ; 19: e3182, jan. - dez. 2018.

OYIBO, N.; WELlER, G.; WATT, J. Avaliação da percepção leiga da mortalidade materna para melhorar a comunicação de risco: um estudo de caso no estado de Rivers, na Nigéria. Saúde Pública Glob ; I2 (6): 666-679, 2017.

PACAGNELLA, R.C.; PEREIRA, N.M.; SPONHOLZ, F.G.; AGUIAR, R.A.L.P.; GUERRA, G.V.Q.L.; DINIZ, C.S.G.; CAMPOS, B.B.N.S ,MORAES FILHO, E.M.A.O. Mortalidade Materna no Brasil: Propostas e Estratégias para a sua redução. Rev Bras Ginecol Obstet.40: 501 - 506; 2018.

PACKER, A.L; TARDELli, A.O.; CASTRO, R.C.F. A distribuição do conhecimento científico público em informação, comunicação e informática em saúde indexado nas bases de dados MEDLINE e LILACS. Ciênc. saúde coletiva vol.12 no.3 Rio de Janeiro, 2017.

PEREIRA, R. S.V.; MUDJALIEB, A.A. The challenge of changing the care model of labor and birth in the Priority Maternity Hospitals in the state of Rio de Janeiro. Divulg. saúde debate ; (54):57-63, mar. 2016.

PÍCOLI, R.P. ; CAZOLA, L.H.O. ; NÓS LEMOS, E.F. Maternal mortality according to race/skin color in Mato Grosso do Sul, Brazil, from 2010 to 2015 Rev. Bras. Saúde Mater. Infantil. (Online) ; i7 (4): 729-737, out.-dez. 2017.

POLLARD, KERRY . Lançamento do relatório MBRRACE-UK em dezembro de 2016. Parteira Prática ; 20 (2): 30-2, 2017.

SCARTON, J.; PAULA, S. F. DE; ANDRADE, G. B. DE; RANGEL, R. F.; VENTURA, J.; SIQUEIRA, H. C. H. DE. Maternal mortality profile: an integrative literature review / El perfil de lamortalidad materna: una revisión integradora 
Rev. pesqui. cuid. fundam. (Online) ; II(3): 816-822, abr.-maio, 2019.

SZWARCWALD,C. L.; et al. Pesquisa Nacional de Saúde no Brasil: concepção e metodologia de aplicação. Ver. Ciências e saúde coletiva. Vol.ı, N 2, pp 333-342, 2014.

TESFAYE， G.; LOXTON， D.H ; CHOJENTA， C. ; ASSEFA， N. ; SMITH， R. . Magnitude, tendências e causas da mortalidade materna entre mulheres em idade reprodutiva no sistema de saúde e vigilância demográfica de Kersa, leste da Etiópia. BMC Saúde da Mulher ; I8 (I): 198, 2018. 